\title{
Was ist uns wichtig?
}

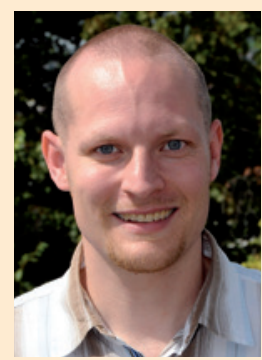

Christian Schäfer, Stuttgart
Eine Frage, die man sich als Person oder auch als Gesellschaft von Zeit zu Zeit stellen sollte, ist diejenige, was denn wirklich zählt und wichtig ist. Bzw. es ist von Vorteil, sich hierüber Gedanken zu machen, um vielleicht die Prioritäten anzupassen oder im besten Fall sich darin bestätigt sehen, dass es eigentlich gerade richtig gut läuft.

Was ist uns nun wichtig? Redet man in Deutschland mit Menschen unterschiedlichen Alters, stellt man fest, dass sich die Prioritäten im Laufe des Lebens verändern. So sind in jungen Jahren des Öfteren folgende Dinge dominant: Materielles, ein reger Kontakt zu möglichst vielen Freunden, Erfahrungen in Partnerschaft und Liebe sammeln, Reisen, Abenteuer, die Wahl der Ausbildung bzw. des Studienfachs, die Karriere usw. Später bleibt zwar je nach Charakter der eine oder andere Faktor in seiner Wichtigkeit bestehen. Es drängen sich aber bei einigen auf der Skala vermehrt der Kontakt zur Familie, Sicherheit, verstärkte Verantwortungsübernahme für die Umwelt und andere Menschen (Kinder, die Eltern etc.), Lebenszufriedenheit, Gesundheit usw. in den Vordergrund. Gerade die Gesundheit wird mit zunehmendem Alter ein immer relevanterer Faktor: So langsam schleicht sich das eine oder andere Zipperlein ein, man merkt, dass es „nicht mehr so wie früher geht“. Wenn man gesundheitlich sehr eingeschränkt ist, kann es abhängig von der Art der Krankheit sehr viel schwerer sein, das Leben zu genießen - selbst wenn man die Zeit dazu hätte.

Wie sieht die Zukunft aus? In einer alternden Gesellschaft, wie die deutsche eine ist, wird sich die Zahl von kranken bzw. multimorbiden Menschen sehr wahrscheinlich weiter erhöhen. Wenn man sich anschaut, wie der deutsche Gesundheitssektor darauf vorbereitet ist, so fällt es einem schwer, sich beruhigt zurückzulehnen: Die derzeitigen Rahmenbedingungen begünstigen es, dass Krankenhäuser sich an Fallzahlen bzw. Wirtschaftlichkeit orientieren, statt die qualitativ hochwertige und adäquate Versorgung der Patienten als absolutes Nummer-1-Kriterium zu etablieren. An teurem und qualifiziertem Personal wird unter ökonomischem Druck eher mal gespart, wenn man es denn im Szenario des Fachkräftemangels überhaupt noch bekommt. Dass dies gefährlich ist, können Sie z.B. in der Rubrik „Journal-Club Pflege“ auf Seite 460 nachlesen.

Patienten werden nach Behandlungen tendenziell eher früher als später aus dem Krankenhaus entlassen, um möglichst zu sparen. Für manche der Patienten kommt dieser Schritt vielleicht zu früh und es treten in diesem Fall eventuell medizinische Komplikationen auf, die vermeidbar gewesen wären. Letztendlich kommt hierbei die Frage auf, ob es nicht besser wäre, die Geldverteilung mehr an anderen Maßstäben festzumachen. Bei einem so wichtigen und grundsätzlichen Gut wie der Gesundheit sollte man Kompromisse so weit wie möglich vermeiden. Belohnen sollte man v.a. eine hohe Behandlungsqualität mit allen dazu gehörigen Faktoren - denn dies ist doch das, um was es letztendlich in Krankenhäusern gehen sollte.

Gerade in einem gemessen am Bruttoinlandsprodukt so reichen Land wie Deutschland sollte die Finanzierung der Gesunderhaltung der Bürger eigentlich kein ernsthaftes Problem sein. Bzw. es weist auf einen Missstand hin, wenn dies hierzulande zu einem Problem zu werden droht. Ganz nebenbei besteht auch noch eine einfache Verknüpfung der Gesundheit der Menschen mit der Leistungsfähigkeit unserer Marktwirtschaft gesunde Bürger, die bis zur Rente arbeiten können, kosten die Gesellschaft weniger und leisten mehr. Aktuell wird die Klinikqualität in der Politik heiß diskutiert und ein neues „Qualitätsinstitut“ wird aufgebaut - schauen wir mal, was sich hier noch tut.

Um die Verbesserung der Gesundheit der in diesem Fall nephrologischen Patienten geht es auch in den Schwerpunktbeiträgen der vorliegenden Ausgabe der Dialyse aktuell: Es ist sehr wichtig, u.a. das kardiovaskuläre Risiko dieser ohnehin meist schon multimorbiden Patientengruppe möglichst niedrig zu halten. Hiermit beschäftigen sich die Artikel ab Seite 462. Ich wünsche Ihnen eine angenehme Lektüre dieser (und auch der anderen) Beiträge der Dialyse aktuell 9/2014! 